\title{
Jozef Nagy
}

Kronecker index in abstract dynamical systems, II

Czechoslovak Mathematical Journal, Vol. 16 (1966), No. 1, 114-126

Persistent URL: http://dml.cz/dmlcz/100715

\section{Terms of use:}

(C) Institute of Mathematics AS CR, 1966

Institute of Mathematics of the Czech Academy of Sciences provides access to digitized documents strictly for personal use. Each copy of any part of this document must contain these Terms of use.

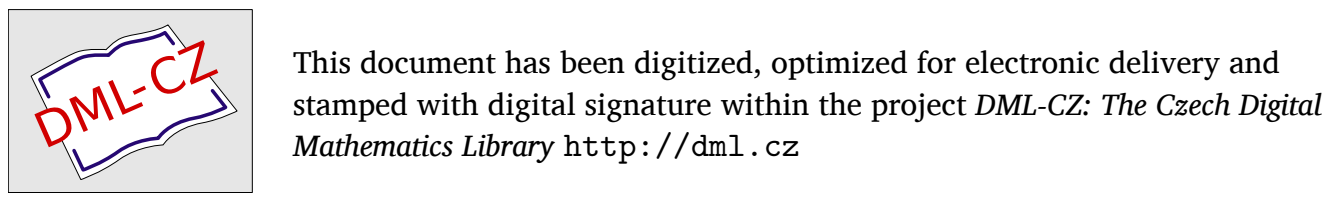




\title{
KRONECKER INDEX IN ABSTRACT DYNAMICAL SYSTEMS, II
}

\author{
JozeF NAGY, Praha
}

(Received February 11, 1965)

In [6] there was defined the index of a point or of a simple loop in $R^{2}$, and then applied to the investigation of qualitative properties of critical points of dynamical systems in $R^{2}$. Now we will attempt to extend this definition to the case of a local dynamical system defined in $p$-dimensional Euclidean space $\mathrm{R}^{p}$. In [6] it was shown that the index of the boundary of a Jordan region in $R^{2}$, under certain suppositions, yields considerable information about the behaviour of the dynamical system within this domain. Particularly interesting are the cases when this boundary is a closed. trajectory or transversal. Of course, these results are characteristic for the case $p=2$ and it seems at a first sight that for $p>2$ the use of indices would not lead to such elegant results. However, it will be shown in the present paper that one may obtain, for general $p$, several interesting results, which are generalisations of results of [6]. These include e.g. the theorem on the index of the boundary of an invariant domain in $R^{p}$, the theorem on the expression of the index of the boundary of a domain by means of the sum of indices of enclosed critical points, etc. The main results of this paper are contained in theorems $2.10,2.12,2.16,2.17$ and 2.18.

2.1. Let us recall some notions and notation. We use throughout the notation introduced in [6]. The results of paper [6] will be referred to directly; e.g. theorem 1.17 is theorem 1.17 in [6]. We suppose as known the notions of an Euclidean $r$-dimensional simplex ( $r$-simplex), of a simplicial complex and its subdivision, of a simplicial map, of a polyhedron and its triangulation, of an $r$-chain of the given complex (over the group $\mathscr{I}_{0}$ of integers) and of the corresponding elementary notions of algebraic topology (see e.g. [2, I, $\S 1]$ ). As coefficient group we use only the group $\mathscr{I}_{0}$ of integers; this assumption will not be repeated.

If $\Pi$ is a triangulation of a polyhedron $P$, we denote by $C_{r}(\Pi)$ the group of $r$-chains, $\partial_{r}$ the boundary operator $C_{r}(\Pi)$ into $C_{r-1}(\Pi) ; Z_{r}(\Pi)$ and $B_{r}(\Pi)$ denote the groups of $r$-cycles and $r$-boundaries respectively; $H_{r}(\Pi)$ denotes the factor group $Z_{r}(\Pi) / B_{r}(\Pi)$ (the $r$-th homology group of the triangulation $\Pi$ ). For a map $f$ of a polyhedron $P$ into a polyhedron $P_{1}$, simplicial with respect to the triangulations $\Pi, \Pi_{1}$ respectively, we denote by $f_{\# r}$ the homomorphism $C_{r}(\Pi) \rightarrow C_{r}\left(\Pi_{1}\right)$ induced by $f$. The corresponding induced homomorphism $H_{r}(\Pi) \rightarrow H_{r}\left(\Pi_{1}\right)$ we denote by $f_{* r}$. 
For a polyhedron $P$ let $H_{r}(P)$ be its $r$-th homology group, for a continuous map $f: P \rightarrow P_{1}$ let $f_{* r}$ be the homomorphism $H_{r}(P) \rightarrow H_{r}\left(P_{1}\right)$ induced by $f$. This twofold use of the symbols $f_{*}$ and $H_{r}$ possibly will not lead to a misunderstanding. We shall write $f_{\#}, f_{*}, \partial$ in place of $f_{\# r}, f_{* r}, \partial_{r}$ whenever the particular value of $r$ is not essential.

2.2. A compact triangulable subset $P$ of the space $\mathrm{R}^{p}$ will be called an $n$-pseudomanifold $(n<p)$ if at least one its triangulations has the following five properties:

(i) $\Pi$ contains at least one $n$-simplex but no $k$-simplex for $k>n$;

(ii) for each two $n$-simplices $\tau, \tau^{\prime}$ of the complex $\Pi$ there exists a sequence $\tau=$ $=\tau_{1}, \tau_{2}, \ldots, \tau_{s}=\tau^{\prime}$ of $n$-simplices of $\Pi$ such that any two neighbouring $n$-simplices of this sequence have an $(n-1)$-side in common;

(iii) each $(n-1)$-simplex of $\Pi$ is a side of precisely two $n$-simplices of $\Pi$;

(iv) each $m$-simplex $(m<n)$ of $\Pi$ is a side of at least one $n$-simplex of $\Pi$;

(v) the pair (Int $P, P$ ), where Int $P$ is a bounded component of $\mathrm{R}^{p}-P$, is a triangulable pair [3].

It may be noted that the notion of a pseudomanifold used in this paper is not equivalent with that used in [1] and [2], where the property (v) is not required.

The set Int $P$ in (v) is determined uniquely, since according to the Jordan-Brouwer Theorem [1, X, $\S 2$, theorem IV], every $(p-1)$-pseudomanifold $P$ in $\mathrm{R}^{p}$ has two complementary domains, of each of which it is the complete frontier, i.e.

$$
\mathrm{R}^{p}-P=\operatorname{Int} P \cup \operatorname{Ext} P,
$$

where Int $P$ (the inner domain of $P$ ) and Ext $P$ (the outer domain of $P$ ) are non-void disjoint sets with common frontier $P$. By definition, the domain Int $P$ is bounded, Ext $P$ unbounded.

An $n$-pseudomanifold will be called orientable if $H_{n}(P)$ is isomorphic to $\mathscr{I}_{0}$. An orientable $n$-pseudomanifold will be called oriented if one of the generators of $H_{n}(P)$ is declared to be orienting. If $Z$ is an orienting generator of $H_{n}(P)$, than each cycle $\zeta \in Z$ will be called an orienting cycle of $P$.

The simplest example of an $n$-pseudomanifold in $R^{p}$ (being at the same time an $n$-manifold, i.e. each of its points has a neighbourhood homeomorphic to $\mathrm{R}_{n}$ ) is the $n$-dimensional sphere $S^{n}$ for $0<n<p$. The groups $H_{0}\left(S^{n}\right)$ and $H_{n}\left(S^{n}\right)$ are isomorphic to $\mathscr{I}_{0}, H_{i}\left(S^{n}\right)$ for $0<i<n$ are trivial $[3, \mathrm{I}$, theorem 16.6]. Hence, the sphere $S^{n}$ is an orientable $n$-pseudomanifold.

According to $[1, \mathrm{X}, \S 2]$ for every $(p-1)$-pseudomanifold $P$ the group $H_{p-1}(P)$ is isomorphic to $\mathscr{I}_{0}$, hence, every $(p-1)$-pseudomanifold in $\mathrm{R}^{p}$ is orientable.

According to $[1, \mathrm{~V}, \S 4.6,(15)]$ for every $p$-dimensional polyhedron $Q$ in $\mathrm{R}^{p}$ the group $H_{n}(Q)$ is isomorphic to the direct sum $\sum_{\pi_{n}(Q)} \mathscr{I}_{0}+\Theta_{n}(Q)$, where $\pi_{n}(Q)$ and $\Theta_{n}(Q)$ denote $n$-th Betti number and $n$-th torsion group of $Q$ respectively. Hence 
there follows that $H_{p-1}($ Int $P)=0$ for every $(p-1)$-pseudomanifold in $\mathrm{R}^{p}$. Indeed, according to $[1, \mathrm{XI}, \S 3.12] \Theta_{p-1}($ Int $P)$ is trivial and from the Alexander Duality Theorem [1, XI, $\S 4.1]$ there follows that $\pi_{p-1}($ Int $P)=\pi_{0}($ Ext $P)-1=0$.

Definition 2.1. Let $P, Q$ be oriented $n$-pseudomanifolds, $Y, Z$ orienting generators of the groups $H_{n}(P), H_{n}(Q)$ respectively, $f: P \rightarrow Q$. The degree of the map $f$ is defined as the unique number $\operatorname{deg}(f)$ satisfying the relation $f_{*} Y=\operatorname{deg}(f) Z$.

The following three propositions are almost trivial:

$(\alpha) \operatorname{deg}(f)$ is an integer.

(B) If $f_{1} \simeq f_{2}$ then $\operatorname{deg}\left(f_{1}\right)=\operatorname{deg}\left(f_{2}\right)$.

$(\gamma)$ On changing the orientation of precisely one of the n-pseudomanifolds $P, Q$, the degree changes sign.

We shall also need to choose coherent orientations for all unit $(p-1)$-spheres in $\mathrm{R}^{p}$. Let $S_{0}^{p-1}$ be the $(p-1)$-sphere in $\mathrm{R}^{p}$ with center 0 and radius 1 . Choosing an orienting generator of $H_{p-1}\left(S_{0}^{p-1}\right)$, we obtain the oriented sphere $S_{0}^{p-1}$. Now define the orienting generator of an arbitrary sphere $S_{a}^{p-1}$ with center $a$ and radius 1 so that $\operatorname{deg}(t)=1$ for the translation $t: S_{a}^{p-1} \rightarrow S_{0}^{p-1}$. By the oriented sphere $S_{a}^{p-1}$ we mean throughout the sphere $S_{a}^{p-1}$ oriented in this manner.

2.3. Now we shall introduce the concept of the order of a point with respect to a map in $R^{p}$.

Definition 2.2. Let $S_{a}^{p-1}$ be an oriented sphere with center in $a, \pi_{a}: \mathrm{R}^{p}-\{a\} \rightarrow$ $\rightarrow S_{a}^{p-1}$ the projection from the point $a, P$ an oriented $(p-1)$-pseudomanifold, $l: P \rightarrow \mathrm{R}^{p}-\{a\}$. The order, $\omega(a, l)$, of a with respect to $l$ is defined as the number $\operatorname{deg}\left(\pi_{a} l\right)$.

In the following lemmas several properties of the order $\omega(a, l)$ will be given, which we shall often use later.

Lemma 2.3. Let $P$ be an oriented $(p-1)$-pseudomanifold, $l, l_{1}, l_{2}: P \rightarrow R^{p}-\{a\}$. Then there hold the following propositions:

(i) if $\pi_{a} l_{1} \simeq \pi_{a} l_{2}$ in $S_{a}^{p-1}$, then $\omega\left(a, l_{1}\right)=\omega\left(a, l_{2}\right)$;

(ii) $\omega(a, l)$ is an integer;

(iii) on changing the orientation of $P$, the order $\omega(a, l)$ changes its sign;

(iv) if $a, a^{\prime}$ are points in the same component of $\mathrm{R}^{p}-l(P)$, then $\omega(a, l)=\omega\left(a^{\prime}, l\right)$.

(The proof is immediate.)

Let $K$ be an arbitrary component of the set $\mathrm{R}^{p}-l(P)$. Proposition (iv) allows one to define the order $\omega(K, l)$ of the component $K$ as the number $\omega(a, l)$, where $a$ is an arbitrary point in $K$. In particular, if $l(P)$ is a $(p-1)$-pseudomanifold, then $\omega(\operatorname{Int} l(P), l)$ and $\omega(\operatorname{Ext} l(P), l)$ are well-defined. 
Lemma 2.4. Let $P$ be an oriented ( $p-1)$-pseudomanifold, $a \in \operatorname{Int} P, i: P \subset$ $\subset \mathrm{R}^{p}-\{a\}$. Then there hold the following propositions:

(i) $\omega($ Int $P, i)=\varepsilon_{P}$, where $\left|\varepsilon_{P}\right|=1$;

(ii) $\omega($ Ext $P, i)=0$; or more generally

(ii) $^{\prime}$ if $l: P \rightarrow \mathrm{R}^{p}-\{0\}, K$ the unbounded component of $\mathrm{R}^{p}-l(P)$, then $\omega(K, l)=0$.

(For the proof see [1], chap. XI, $\S 4$, theorem III.)

In the situation of lemma 2.1 , if $\varepsilon_{P}=1$, then we shall say that the $(p-1)$-pseudomanifold $P$ is positively oriented; the corresponding orienting generator (or cycle) will be called a positive orienting generator (cycle); in the opposite case the $(p-1)$ pseudomanifold will be called negatively oriented and we shall speak about a negative orienting generator (cycle).

Lemma 2.5. Let $P$ be an oriented $(p-1)$-pseudomanifold, $f: \mathbf{R}^{p} \approx \mathbf{R}^{p}, a \in \mathbf{R}^{p}$, $f_{0}: \mathrm{R}^{p}-\{a\} \approx \mathrm{R}^{p}-\{f(a)\}: f_{0}(x)=f(x), l: P \rightarrow \mathrm{R}^{p}-\{a\}, i: S_{a}^{p-1} \subset \mathrm{R}^{p}-\{a\}$ Then $\omega\left(f(a), f_{0} l\right)=\omega\left(f(a), f_{0} i\right) . \omega(a, l)$ and $\left|\omega\left(f(a), f_{0} i\right)\right|=1$.

Proof. Let $S, S_{1}, Z$ be orienting generators of $H_{p-1}\left(S_{a}^{p-1}\right), H_{p-1}\left(S_{f(a)}^{p-1}\right), H_{p-1}(P)$ respectively, and $\pi: \mathrm{R}^{p}-\{a\} \rightarrow S_{a}^{p-1}, \pi_{1}: \mathrm{R}^{p}-\{f(a)\} \rightarrow S_{f(a)}^{p-1}$ projections. From the definition 2.1 there follow (in the $(p-1)$-st homological groups)

$$
(\pi l)_{*} Z=\omega(a, l) S, \quad\left(\pi_{1} f_{0} l\right)_{*} Z=\omega\left(f(a), f_{0} l\right) S_{1}, \quad\left(\pi_{1} f_{0} i\right)_{*} S=\omega\left(f(a), f_{0} i\right) S_{1} .
$$

Define a homotopy $h_{\lambda}: i \pi l \simeq l$ in $\mathrm{R}^{p}-\{a\}$ as follows: $h_{\lambda}(x)=(1-\lambda) i \pi l(x)+$ $+\lambda l(x)$. Clearly, $\pi_{1} f_{0} h_{\lambda}$ is then a homotopy $\pi_{1} f_{0} i \pi l \simeq \pi_{1} f_{0} l$ in $S_{f(a)}^{p-1}$, and hence $\left(\pi_{1} f_{0} i \pi l\right)_{*} Z=\left(\pi_{1} f_{0} l\right)_{*} Z$. Now, there is

$$
\begin{aligned}
& \omega\left(f(a), f_{0} l\right) S_{1}=\left(\pi_{1} f_{0} l\right)_{*} Z=\left(\pi_{1} f_{0} i \pi l\right)_{*} Z=\left(\pi_{1} f_{0} i\right)_{*}(\pi l)_{*} Z= \\
= & \left(\pi_{1} f_{0} i\right)_{*} \omega(a, l) S=\omega(a, l)\left(\pi_{1} f_{0} i\right)_{*} S=\omega(a, l) . \omega\left(f(a), f_{0} i\right) S_{1},
\end{aligned}
$$

and thus finally

$$
\omega\left(f(a), f_{0} l\right)=\omega\left(f(a), f_{0} i\right) \cdot \omega(a, l) .
$$

For the proof of the relation $\left|\omega\left(f(a), f_{0} i\right)\right|=1$ see [1], chap. XII, $\S 2$, theorem VI.

The lemma 2.5 allows one to assign, to every homeomorphism $f: \mathrm{R}^{p} \approx \mathrm{R}^{p}$, the number $e_{f}=\omega\left(f(o), f_{0} i\right)$; the homeomorphism $f$ is said to be orientation preserving if $e_{f}=1$, and orientation reversing if $e_{f}=-1$.

Lemma 2.6. Let $s y m_{0}: \mathrm{R}^{p} \approx \mathrm{R}^{p}$ be the symmetry of $\mathrm{R}^{p}$ relative to the origin, (i.e. the affine mapping given by the matrix $\left(a_{j k}\right), a_{j j}=-1, a_{j k}=0$ for $j \neq k$, $j, k=1,2, \ldots, n)$; then $e_{\text {symo }}=(-1)^{p}$. More generally, if sym: $\mathbf{R}^{p} \approx \mathrm{R}^{p}$ is a symmetry of $\mathrm{R}^{p}$ relative to a point $x_{0}$, and $S$ an oriented sphere with center in $x_{0}$, $i: S \subset \mathrm{R}^{p}, f_{0}: S \rightarrow \mathrm{R}^{p}-\left\{x_{0}\right\}: f_{0}(x)=\operatorname{sym} i(x)$, then $\omega\left(x_{0}, f_{0}\right)=(-1)^{p} \cdot \varepsilon_{S}$. 
Proof. Clearly $e_{s y m_{0}}=\operatorname{det}\left(a_{j k}\right)=(-1)^{p}$ and $\omega\left(x_{0}, f_{0}\right)=\varepsilon_{s} . e_{s y m_{0}}$.

2.4. In this paragraph we shall introduce the notions of a local dynamical system [4] and of vector fields associated with a local dynamical system, which will play a very important role in the remaining part of the paper.

Definition 2.7. A local dynamical system on a topological space $\mathrm{P}$ is a partial mapping $T$ out of $P \times R^{1}$ into $P$ which satisfies the following five conditions:

(1) the domain of $T$ is open;

(2) $T$ is continuous;

(3) to each $x \in \mathrm{P}$ there exist $\alpha_{x}, \beta_{x}$ with $-\infty \leqq \beta_{x}<0<\alpha_{x} \leqq+\infty$ and such that $x \mathrm{~T} \theta$ is defined iff $\beta_{x}<\theta<\alpha_{x}$, where $x \mathrm{~T} \theta$ denotes the value of the mapping $\mathrm{T}$ at the point $(x, \theta) \in \mathrm{P} \times \mathrm{R}^{1}$, if this is defined;

(4) $x \mathrm{~T} 0=x$ obtains for all $x \in \mathrm{P}$;

(5) $\left(x \mathrm{~T} \theta_{1}\right) \mathrm{T} \theta_{2}=x \mathrm{~T}\left(\theta_{1}+\theta_{2}\right)$ holds if both $x \mathrm{~T} \theta_{1}$ and also the left or right side of this relation are defined.

A local dynamical system with $\mathrm{P} \times \mathrm{R}^{1}$ as domain will be termed a global dynamical system.

For $\mathrm{R} \subset \mathrm{P}, A \subset \mathrm{R}^{1}$ denote $X \mathrm{~T} A=\{x \mathrm{~T} \theta: x \in X, \theta \in A, x \mathrm{~T} \theta$ defined $\}$. Let $\mathrm{T}$ be a local dynamical system in $P$. A point $x \in P$ is said to be a critical point of the local dynamical system $\mathrm{T}$ if $x \mathrm{~T} \theta=x$ holds for all $\theta$ such that $x \mathrm{~T} \theta$ is defined. If there exists a least $\theta_{0}>0$ such that $x \mathrm{~T} \theta_{0}=x$ holds, then the set $x \mathrm{TR}^{1}$ will be called a periodic trajectory with the primitive period $\theta_{0}$. Every number $k \theta_{0}$ with $k$ an integer will be called a period of the periodic trajectory $x \mathrm{TR}^{1}$. If $x \mathrm{~T} \theta \neq x$ holds for all $\theta \in \mathbf{R}^{1}$ such that $x \mathrm{~T} \theta$ is defined, then the set $x \mathrm{~T}^{1}$ will be called a (non-periodic) trajectory.

A set $X \subset \mathrm{P}$ is termed a +invariant (or -invariant, invariant) set of the local dynamical system $\mathrm{T}$ if $X \mathrm{~T}\langle 0,+\infty) \subset X, \quad\left(\right.$ or $X \mathrm{~T}(-\infty, 0\rangle \subset X, \quad X \mathrm{TR}^{1} \subset X$ respectively).

Now consider a local dynamical system $\mathrm{T}$ on $\mathrm{R}^{p}$, and let $K \subset R^{p}, 0<\varepsilon \in \mathrm{R}^{1}$, $\vartheta: K \rightarrow(0, \varepsilon)$ any continuous mapping such that $x \mathrm{~T} \vartheta(x)$ is defined for all $x \in K$. A vector field of the local dynamical system $\mathrm{T}$ on $K$ is the mapping $W: K \rightarrow \mathrm{R}^{p}$ defined by

$$
W(x)=x \operatorname{T} \vartheta(x)-x
$$

The mapping $W$ is continuous. Clearly, $W$ vanishes at a point $x_{0}$ if either $x_{0}$ is a critical point of the local dynamical system $\mathrm{T}$, or $x_{0}$ is on a periodic trajectory with the period $\vartheta\left(x_{0}\right)$, or $\vartheta\left(x_{0}\right)=0[5, \mathrm{VI}, \S 1]$.

If $\vartheta_{1} \simeq \vartheta_{2}$, then, obviously, the corresponding vector fields are also homotopic. 
Lemma 2.8. Let $\mathrm{T}$ be a local dynamical system in $\mathrm{R}^{p}, F \subset \mathrm{R}^{p}$ compact non-void and containing no critical point of $\mathrm{T}$. Then there exists an $A, 0<A \in \mathrm{R}^{1}$, such that

(i) $x \mathrm{~T} \theta$ is defined for every $(x, \theta) \in \mathrm{R}^{p} \times\langle 0, A\rangle$;

(ii) for every mapping $\vartheta: F \rightarrow(0, A)$, the mapping $W$ defined by (1) is a vector field of the local dynamical system $\mathrm{T}$, continuous and vanishing nowhere on $F$.

Proof. Let $x \in F$. There exists an $\alpha_{x}>0$ such that $x \mathrm{~T} \theta$ is defined for every $\theta \in\left\langle 0, \alpha_{x}\right)$. The domain of $\mathrm{T}$ is open; hence there exists a neighbourhood $G_{x}$ of $x$ and an $a_{x} \in \mathrm{R}^{1}$ such that $0<a_{x}<\alpha_{x}$ and $y \mathrm{~T} \theta$ is defined for every $(y, \theta) \in G_{x} \times$ $\times\left\langle 0, a_{x}\right)=H_{x}$. The set $H_{x}$ is open in $\mathrm{R}^{p} \times\langle 0,+\infty)$. Clearly, the system $\left\{H_{x}\right\}_{x \in F}$ is an open cover of $F \times\{0\}$; then there is a finite cover $\left\{H x_{i}\right\}_{i=1}^{r} \subset\left\{H_{x}\right\}_{x \in F}$. Now assertion (i) holds for every $A$ with $0<A<\min \left\{a_{x_{1}}, a_{x_{2}}, \ldots, a_{x_{r}}\right\}$.

The proof of (ii) is the same as that of lemma 1.5 in [6].

Let now T, $F, A$ be as in lemma 2.8. Then for every two mappings $\vartheta_{0}, \vartheta_{1}: F \rightarrow$ $\rightarrow(0, A)$ there exists a homotopy $\vartheta_{\lambda}: \vartheta_{0} \simeq \vartheta_{1}$ (e.g. $\vartheta_{\lambda}(x)=(1-\lambda) \vartheta_{0}(x)+$ $\left.+\lambda \vartheta_{1}(x)\right)$ such that, for every $\lambda \in I$, the mapping $W_{\lambda}$ defined as in (1) is a vector field of the system $\mathrm{T}$, continuous and vanishing nowhere on $F$. Every vector field $W$ on $F$ defined by (1) with $\vartheta: F \rightarrow(0, A)$ defines a mapping $W_{0}: F \rightarrow \mathrm{R}^{p}-$ $-\{0\}: W_{0}(x)=W(x)$. Every such mapping $W_{0}$ will be termed a small vector field of the local dynamical system $\mathrm{T}$ on $F$.

2.5. From lemma 2.8 it follows that, for every $(p-1)$-pseudomanifold $P$ in $\mathbf{R}^{p}$ containing no critical points of the local dynamical system $\mathrm{T}$, there exist small vector fields on $P$. If $W_{1}, W_{2}$ are two small vector fields on $P$, then $0 \notin W_{1}(P) \cup W_{2}(P)$, and according to lemmas 2.8 and $2.2(\mathrm{i})$, there holds $\omega\left(0, W_{1}\right)=\omega\left(0, W_{2}\right)$. Now we can set up the following definition.

Definition 2.9. Let $\mathrm{T}$ be a local dynamical system in $\mathrm{R}^{p}, P$ an oriented $(p-1)$ pseudomanifold in $\mathrm{R}^{p}$ containing no critical points of $\mathrm{T}, W$ a small vector field on $P$. The Kronecker index ind $P$ of $P$ relative to $\mathrm{T}$ is defined as $\varepsilon_{P} . \omega(0, W)$.

Immediately from the definition it follows that $\operatorname{ind}_{\mathrm{T}} P$ is an integer not depending on the orientation of $P$; thus in this definition the assumption on the orientation of $P$ can be weakened to orientability only.

Theorem 2.10. Let $P$ be a $(p-1)$-pseudomanifold in $\mathrm{R}^{p}, \mathrm{~T}$ a local dynamical system in $\mathrm{R}^{p}$ without critical points in Int $P$. Then $\operatorname{ind}_{\mathrm{T}} P=0$.

Proof. Let $Q=\overline{\operatorname{Int} P}, i: P \subset Q, Z$ an orienting generator of $H_{p-1}(P), S$ an orienting generator of $H_{p-1}\left(S_{0}^{p-1}\right)$. It is easily shown that $H_{p-1}(Q)=0$. Hence it follows that $i_{*} Z$ is the zero element of $H_{p-1}(Q)$. According to lemma 2.8 there exists a small vector field $W$ on $Q$, hence for $\pi W: Q \rightarrow S_{0}^{p-1}$ there holds

$$
(\pi W)_{*} Z=0 \text {. }
$$


Clearly, $W i$ is a small vector field on $P$, and from (2) and the definition 2.1 one obtains $0=(\pi W i)_{*} Z=\omega(0, W i) S$; it follows that $\omega(0, W i)=0$ and thus $\operatorname{ind}_{\mathrm{T}} P=\varepsilon_{P}$. . $\omega(0, W i)=0$.

Corollary 2.11. If ind $_{\mathrm{T}} P \neq 0$, then Int $P$ contains at least one critical point of the local dynamical system $\mathrm{T}$.

Theorem 2.12. Let $\mathrm{T}$ be a local dynamical system on $\mathrm{R}^{p}, S$ a $(p-1)$-sphere containing no critical point of $\mathrm{T}$. Then

(i) $\operatorname{ind}_{\mathrm{T}} S=1$ if Int $S$ is -invariant,

(ii) ind $_{\mathrm{T}} S=(-1)^{p}$ if Int $S$ is +invariant.

Proof. Denote by $x_{0}$ and $r$ the center and the radius of the sphere $S, i: S \subset R^{p}-$ $-\left\{x_{0}\right\}$.

Ad (i). From the assumptions it follows that there exists an $A>0$ such that $\left|x \mathrm{~T} \theta-x_{0}\right| \geqq r$ and $x \mathrm{~T} A \neq x$ for all $(x, \theta) \in S \times\langle 0, A\rangle$. Define mappings $W_{0}, W_{1}$ : $: S \rightarrow R^{p}-\{0\}$ and a homotopy $W_{\lambda}: W_{0} \simeq W_{1}$ as follows:

$$
\begin{gathered}
W_{0}(x)=x-x_{0}, \quad W_{1}(x)=x \mathrm{~T} A-x, \\
W_{\lambda}(x)=x \mathrm{~T}(\lambda A)-x_{0}-\lambda\left(x-x_{0}\right) .
\end{gathered}
$$

Obviously, $W_{\lambda}$ is continuous and vanishes nowhere on $S$, since $\left|x T(\lambda A)-x_{0}\right| \geqq$ $\geqq r>\lambda\left|x-x_{0}\right|$ for $0 \leqq \lambda<1, W_{1}(x)=x \mathrm{~T} A-x \neq 0$. Thus

$$
\omega\left(0, W_{0}\right)=\omega\left(0, W_{1}\right)=\omega\left(x_{0}, i\right)=\varepsilon_{S},
$$

and hence $\operatorname{ind}_{\mathrm{T}} S=\varepsilon_{S} \cdot \omega\left(0, W_{1}\right)=\varepsilon_{S} \cdot \varepsilon_{S}=1$.

Ad (ii): From the assumptions it follows that Int $S$ is also + invariant, so that there exists an $A>0$ such that $\left|x T \theta-x_{0}\right| \leqq r$ for every $(x, \theta) \in S \times\langle 0, A\rangle$. Define mappings $W_{0}, W_{1}: S \rightarrow R^{p}-\{0\}$ and a homotopy $W_{\lambda}: W_{0} \simeq W_{1}$ as follows: $W_{0}(x)=x_{0}-x, W_{1}(x)=x \top A-x, W_{\lambda}(x)=\lambda\left(x \top A-x_{0}\right)+\left(x_{0}-x\right)$. Obviously, $W_{\lambda}$ is continuous and vanishes nowhere on $S$, since $\lambda|x \mathrm{~T} A-x|<r=\left|x_{0}-x\right|$ for $0 \leqq \lambda<1, W_{1}(x)=x \mathrm{~T} A-x \neq 0$. According to lemmas 2.3 and 2.6

$$
\omega\left(0, W_{0}\right)=\omega\left(0, W_{1}\right)=\omega\left(x_{0}, f_{0}\right)=\varepsilon_{S} \cdot(-1)^{p},\left(f_{0}\right. \text { from 2.6), }
$$

and hence

$$
\operatorname{ind}_{\mathrm{T}} S=\varepsilon_{S} \cdot \varepsilon_{S} \cdot(-1)^{p}=(-1)^{p} .
$$

The theorem 2.12 is thus proved.

From (3) and (4) it may be noted that ind $_{\mathrm{T}} S$ in the theorem is expressed by topological invariants $\left(\omega\left(x_{0}, i\right)\right.$ and $\omega\left(x_{0}, s y m\right)$ respectively, see lemma 2.6$)$. The assumption about $S$ in the theorem may now be weakened thus: $S$ is an arbitrary $(p-1)$-manifold in $\mathrm{R}^{p}$, homeomorphic to a $(p-1)$-sphere. 
Corollary 2.13. Let $R^{p}$ be odd-dimensional, let a $(p-1)$-manifold $S$ be homeomorphic to a $(p-1)$-sphere. Then Int $S$ is not an invariant set of any local dynamical system without critical points on $S$.

Proof. Let Int $S$ be the invariant set of a local dynamical system T. Then Int $S$ is also -invariant. From the proposition 2.12 (i) there follows ind $_{\mathrm{T}} S=1$, from (ii) there follows ind $_{\mathrm{T}} S=-1$; this is a contradiction.

Corollary 2.14. If the $(p-1)$-sphere $S$ satisfies the assumptions of the theorem 2.12 , then Int $S$ contains at least one critical point of the local dynamical system $\mathrm{T}$.

For proof see corollary 2.11 .

2.6. Definition 2.9 generalises the definition of the index of a simple loop [6, def. 1.6], and also allows one to generalise in the same manner the notion of the index of a critical point. We shall prove that this generalised index of a critical point is also a topological invariant.

Definition 2.15. Let $\mathrm{T}$ be a local dynamical system, $x \in \mathrm{R}^{p}$ not an accumulation point of critical points of T, $S$ an oriented $(p-1)$-sphere with center $x$ and small enough to have Int $S-\{x\}$ contain no critical points of T. The Kronecker index ind $_{\mathrm{T}} x$ of the point $x$ relative to $\mathrm{T}$ is defined as the number $\operatorname{ind}_{\mathrm{T}} S$.

It is easily shown that ind $\mathrm{T}_{\mathrm{T}} x$ does not depend on the choice of $S$.

By a generalisation of theorem 1.17 we now obtain an important result which also enables us to replace a $(p-1)$-sphere $S$ in definition 2.15 by an arbitrary $(p-1)$ pseudomanifold.

Theorem 2.16. Let the inner domain of a $(p-1)$-pseudomanifold P contain only a finite number $x_{1}, x_{2}, \ldots, x_{n}$ of critical points of a local dynamical system $\mathrm{T}$, and let there be none on $P$. Then

$$
\operatorname{ind}_{\mathrm{T}} P=\sum_{j=1}^{n} \operatorname{ind}_{\mathrm{T}} x_{j} \text {. }
$$

Proof. For $j=1,2, \ldots, n$ let $S_{j}$ be a $(p-1)$-sphere with center in $x_{j}$, such that $S_{k} \cap S_{j}=\emptyset$ holds for $1 \leqq k<j \leqq n$. Denote $Q=\operatorname{Int} P-\bigcup_{j=1}^{n} \operatorname{Int} S_{j}$, $i_{0}: P \subset Q, i_{j}: S_{j} \subset Q$. Let $Z_{0}, Z_{1}, \ldots, Z_{n}$ be positive orienting generators of $H_{p-1}(P), H_{p-1}\left(S_{1}\right), \ldots, H_{p-1}\left(S_{n}\right), Z_{l}^{\prime}=\left(i_{l}\right)_{*} Z_{l}$ for $l=0,1, \ldots, n$.

Let $\pi: \mathrm{R}^{p}-\{0\} \rightarrow S_{0}^{p-1}$ be the projection, $S$ an orienting generator of $S_{0}^{p-1}$. Then it is easily shown that

$$
Z_{0}^{\prime}-\sum_{j=1}^{n} Z_{j}^{\prime}=0
$$


Let $W$ be a small vector field on $Q$. Then from (8) there follows $\underset{n}{(\pi W})_{*}\left(Z_{0}^{\prime}-\sum_{j=1}^{n} Z_{j}^{\prime}\right)=$ $=0$; substituting $Z_{j}^{\prime}=\left(i_{j}\right)_{*} Z_{j}$, one obtains $(\pi W)_{*}\left(i_{0}\right)_{*} Z_{0}=\sum_{j=1}^{n}(\pi W)_{*}\left(i_{j}\right)_{*} Z_{j}$, or, $\underset{n}{\text { equivalently, }} \omega\left(0, W i_{0}\right) S=\sum_{j=1}^{n} \omega\left(0, W i_{j}\right) S ;$ hence there follows $\omega\left(0, W i_{0}\right)=$ $=\sum_{j=1}^{n} \omega\left(0, W i_{j}\right)$. According to the assumption, $P, S_{1}, \ldots, S_{n}$ are positively oriented, i.e. $\varepsilon_{P}=\varepsilon_{S_{1}}=\ldots=\varepsilon_{S_{n}}=1$, so that

$$
\operatorname{ind}_{T} P=\varepsilon_{P} \cdot \omega\left(0, W i_{0}\right)=\sum_{j=1}^{n} \varepsilon_{S_{j}} \cdot \omega\left(0, W i_{j}\right)=\sum_{j=1}^{n} \operatorname{ind}_{\top} x_{j} .
$$

The theorem 2.16 is proved.

From theorem 2.16 it follows immediately that if $x_{0} \in \mathrm{R}^{p}$ is not an accumulation point of critical points of $\mathrm{T}$ and $P_{1}, P_{2}$ are $(p-1)$-pseudomanifolds such that $x_{0} \in$ Int $P_{1} \cap \operatorname{Int} P_{2}, \overline{\text { Int } P_{1}} \cup \overline{\text { Int } P_{2}}-\left\{x_{0}\right\}$ contains no critical point of $\mathrm{T}$, then ind $_{\mathrm{T}} P_{1}=\operatorname{ind}_{\mathrm{T}} P_{2}=\operatorname{ind}_{\mathrm{T}} x_{0}$. Hence the sphere $S$ in definition 2.15 may be replaced by an arbitrary $(p-1)$-pseudomanifold $P$ having the properties described in this definition.

From the definition of local dynamical systems one has directly the following proposition: If $\mathrm{T}$ is a local dynamical system in $\mathrm{R}^{p}, f: \mathrm{R}^{p} \approx \mathrm{R}^{p}$, then the relation $f(x \mathrm{~T} \theta)=f(x) \mathrm{T}_{f} \theta$ defines a local dynamical system $\mathrm{T}_{j}$ in $\mathrm{R}^{p}$. Naturally there arises the question as to the relation between $\operatorname{ind}_{\mathrm{T}} x$ and $\operatorname{ind}_{\mathrm{T}_{f}} f(x)$. The answer is the following theorem on the topological invariance of the index of a point.

Theorem 2.17. Let $x_{0} \in \mathrm{R}^{p}$ not be an accumulation point of critical points of a local dynamical system $\mathrm{T}$ in $\mathrm{R}^{p}, f: \mathrm{R}^{p} \approx \mathrm{R}^{p}$. Then $\operatorname{ind}_{\mathrm{T}} x_{0}=\operatorname{ind}_{\mathrm{T}_{f}} f\left(x_{0}\right)$.

Proof. Let $S$ be as in definition 2.15. Let $\theta \in \mathrm{R}^{1}$ be such that the mapping $W_{0}: S \rightarrow \mathrm{R}^{p}-\{0\}$ given by the relation $W_{0}(x)=x \mathrm{~T} \theta-x$ is a small vector field on $S$. Let $f_{0}: S \rightarrow \mathrm{R}^{p}-\{0\}$ be given by the relation $f_{0}(x)=x \mathrm{~T} \theta$. The proof of the theorem will be divided into two parts.

I. Let $f_{0}(S) \subset$ Ext $S$.

Define a mapping $W_{1}: S \rightarrow \mathrm{R}^{p}-\{0\}$ and a homotopy $W_{\lambda}: W_{0} \simeq W_{1}$ in $\mathrm{R}^{p}-\{0\}$ by the relations (also see fig. 1) $W_{1}(x)=f_{0}(x)-x_{0}, W_{\lambda}(x)=f_{0}(x)-x+\lambda\left(x-x_{0}\right)$. $W_{\lambda}$ is continuous, and vanishes nowhere on $S$, as $W_{\lambda}(x)=f_{0}(x)-x_{0}+(1-\lambda)$. . $\left(x_{0}-x\right),(1-\lambda)\left|x-x_{0}\right|=(1-\lambda) r \leqq r<\left|f_{0}(x)-x_{0}\right|$. Thus according to lemma $2.3, \omega\left(0, W_{0}\right)=\omega\left(0, W_{1}\right)$. From the definition of the mapping $W_{1}$ it follows easily that $\omega\left(x_{0}, f_{0}\right)=\omega\left(0, W_{1}\right)$, so that

$$
\omega\left(0, W_{0}\right)=\omega\left(x_{0}, f_{0}\right),
$$

and hence $\operatorname{ind}_{\mathrm{T}} x_{0}=\varepsilon_{S} \cdot \omega\left(x_{0}, f_{0}\right)$. From lemma 2.5 one obtains $\varepsilon_{f(S)}=e_{f} \cdot \varepsilon_{S}$, $\omega\left(f\left(x_{0}\right), f f_{0}\right)=e_{f} \cdot \omega\left(x_{0}, f_{0}\right)$ and thus ind $\operatorname{T}_{f} f\left(x_{0}\right)=\varepsilon_{f(S)} \cdot \omega\left(f\left(x_{0}\right), f f_{0}\right)=\varepsilon_{S} \cdot e_{f}$. . $e_{f} \cdot \omega\left(x_{0}, f_{0}\right)=\operatorname{ind}_{\mathrm{T}} x_{0}$. The topological invariance of the index in case $I$ is proved. 
II. Let $f_{0}(S) \nsubseteq$ Ext $S$.

First we shall prove one proposition which will make it possible to carry over the proof of case I to the present case.

Proposition. There exist $f_{1}: S \rightarrow \mathrm{R}^{p}-\{0\}, f_{1}(S) \subset$ Ext $S$ and a homotopy $h_{\lambda}: f_{0} \simeq f_{1}$ such that $h_{\lambda}(x) \neq x$ for every $\lambda \in I$.

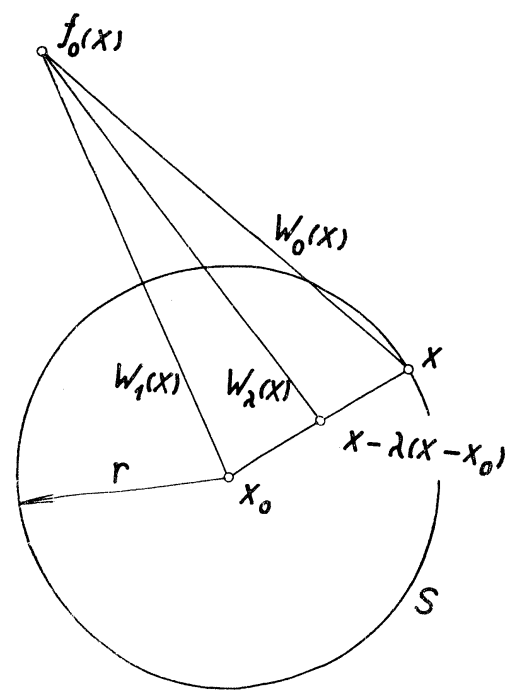

Fig. 1.

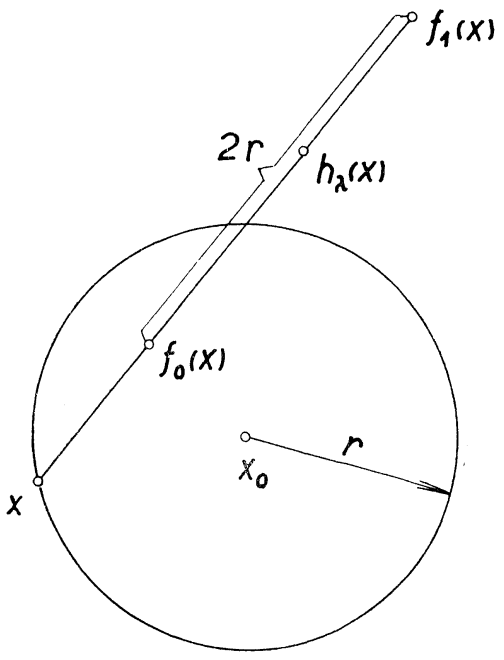

Fig. 2.

Pro of of proposition. Define $f_{1}$ and $h_{\lambda}$ as follows (see fig. 2):

$$
\begin{gathered}
f_{1}(x)=x+\left(\left|f_{0}(x)-x\right|+2 r\right) \frac{f_{0}(x)-x}{\left|f_{0}(x)-x\right|}, \\
h_{\lambda}(x)=x+\left(\left|f_{0}(x)-x\right|+\lambda .2 r\right) \frac{f_{0}(x)-x}{\left|f_{0}(x)-x\right|} .
\end{gathered}
$$

From the relation $\left|f_{1}(x)-x\right|=\left|f_{0}(x)-x\right|+2 r>2 r$ there follows $f_{1}(S) \subset$ Ext $S$, and from the relation $\left|h_{\lambda}(x)-x\right|=\left|f_{0}(x)-x\right|+\lambda .2 r>0$ for $\lambda \in I$ there follows $h_{\lambda}(x) \neq x$ for every $\lambda \in I$.

The proposition is proved.

Returning to the proof of the theorem, we shall show that the mapping $f_{1}$ from the proposition has the properties of the mapping $f_{0}$ from case I. The relation $f_{1}(S) \subset$ $\subset$ Ext $S$ is evident. Defining the mapping $W_{0}^{\prime}: S \rightarrow \mathrm{R}^{p}-\{0\}$ and the homotopy 
$W_{\lambda}: W_{0} \simeq W_{0}^{\prime}$ by $W_{0}^{\prime}(x)=f_{1}(x)-x, W_{\lambda}(x)=h_{\lambda}(x)-x$, we obtain $\omega\left(0, W_{0}\right)=$ $=\omega\left(0, W_{0}^{\prime}\right)$. Furthermore, from $f_{1}(S) \subset$ Ext $S$ there follows (see (6)) $\omega\left(0, W_{0}^{\prime}\right)=$ $=\omega\left(x_{0}, f_{1}\right)$, hence

$$
\omega\left(0, W_{0}\right)=\omega\left(0, W_{0}^{\prime}\right)=\omega\left(x_{0}, f_{1}\right)
$$

This shows that $f_{1}$ has the property of $f_{0}$ from (6). Now we may apply the first part of the proof which concludes the proof of theorem 2.16 .

Now we shall give two results which may be useful for the calculation of the index.

Corollary 2.12. Let the inner domain of a $(p-1)$-pseudomanifold $P$ contain only a finite number of critical points of a local dynamical system $\mathrm{T}$, and let there be none on $P$; let $f: \mathrm{R}^{p} \approx \mathrm{R}^{p}$. Then $\operatorname{ind}_{\mathrm{T}} P=\operatorname{ind}_{\mathrm{T}_{f}} f(P)$.

Proof. If $x_{1}, x_{2}, \ldots, x_{n}$ are all the critical points of $\mathrm{T}$ in Int $P$, then $f\left(x_{1}\right), f\left(x_{2}\right), \ldots$, $\ldots, f\left(x_{n}\right)$ are all the critical points of $\mathrm{T}_{f}$ in Int $f(P)$. From theorems 2.16 and 2.17 there follows ind $P=\sum_{j=1}^{n}$ ind $_{\mathrm{T}} x_{j}=\sum_{j=1}^{n}$ ind $_{\mathrm{T}_{f}} f\left(x_{j}\right)=\operatorname{ind}_{\mathrm{T}_{f}} f(P)$.

Theorem 2.19. Let the inner domain of a $(p-1)$-pseudomanifold P contain only a finite number of critical points of a local dynamical system $\mathrm{T}$ and let there be none on $P$. Let $W_{0}$ be a small vector field on $P$ and let $f: P \rightarrow R^{p}$ be such that the mapping $W_{1}: P \rightarrow \mathrm{R}^{p}-\{0\}$ defined by $W_{1}(x)=f(x)-x$ satisfies on $P$ the relation $\left|W_{0}(x)-W_{1}(x)\right|<\left|W_{0}(x)\right|$. Then $\operatorname{ind}_{\mathrm{T}} P=\varepsilon_{P} . \omega\left(0, W_{1}\right)$.

Proof. We shall prove that $W_{0}$ and $W_{1}$ are homotopic in $R^{p}-\{0\}$. Define a homotopy $W_{\lambda}: W_{0} \simeq W_{1}$ in $\mathrm{R}^{p}-\{0\}$ by $W_{\lambda}(x)=(1-\lambda) W_{0}(x)+\lambda W_{1}(x)$. $W_{\lambda}$ is evidently continuous. We shall prove that it vanishes nowhere on $\mathrm{R}^{p}-\{0\}$. Suppose that there exists a point $\left(\lambda_{0}, x_{0}\right) \in\langle 0,1\rangle \times P$ such that $W_{\lambda_{0}}\left(x_{0}\right)=0$. Then $\left(1-\lambda_{0}\right) W_{0}\left(x_{0}\right)+\lambda_{0} W_{1}\left(x_{0}\right)=0$, hence $W_{0}\left(x_{0}\right)=\lambda_{0}\left(W_{0}\left(x_{0}\right)-W_{1}\left(x_{0}\right)\right)$, and thus $\left|W_{0}\left(x_{0}\right)\right| \leqq\left|W_{0}\left(x_{0}\right)-W_{1}\left(x_{0}\right)\right|$; this contradicts the assumption of the theorem. Thus $W_{\lambda}$ is indeed a homotopy $W_{0} \simeq W_{1}$ in $\mathrm{R}^{p}-\{0\}$, and then the theorem follows immediately.

2.7. In this last paragraph we make one remark concerning the rôle of the unicity of trajectories assumption in our investigation. If $\mathrm{T}$ is a local dynamical system, then the relation $x \mathrm{~T} \theta=y \mathrm{~T} \theta$ implies $x=y$. It may be noted that we have not exploited this property of $T$ in our treatment. Thus it is very natural to generalise, in a certain sense, our results to the category of the so-called local semi-dynamical systems on $R^{p}$ [4]. Let us recall the definition of a local semi-dynamical system $\left(R^{+}\right.$denotes the set of all non-negative reals). 
Definition 2.20. A local semi-dynamical system on a topological space $\mathrm{P}$ is a partial mapping $T$ out of $P \times R^{+}$into $P$ which satisfies the following five conditions:

(1) the domain of $T$ is open;

(2) $T$ is continuous;

(3) to each $x \in \mathrm{P}$ there exists an $\alpha_{x}$ with $0<\alpha_{x} \leqq+\infty$ and such that $x \mathrm{~T} \theta$ is defined iff $0 \leqq \theta<\alpha_{x}$; here $x \mathrm{~T} \theta$ denotes, whenever defined, the value of the mapping $\mathrm{T}$ at the point $(x, \theta) \in \mathrm{P} \times \mathrm{R}^{+}$;

(4) $x \mathrm{~T} 0=x$ obtains for all $x \in \mathrm{P}$;

(5) $\left(x \mathrm{~T} \theta_{1}\right) \mathrm{T} \theta_{2}=x \mathrm{~T}\left(\theta_{1}+\theta_{2}\right)$ holds iff the left or right side of this relation is defined.

A local semi-dynamical system with $\mathrm{P} \times \mathrm{R}^{+}$as domain will be termed a global semi-dynamical system.

It is clear that for a local semi-dynamical system -unicity need not hold, i.e. from the relation $x \mathrm{~T} \theta=y \mathrm{~T} \theta$ there need not follow $x=y$.

The main apparatus in the present paper were small vector fields of local dynamical systems $T$ on compact sets in $R^{p}$. In the definition of these fields only the values of $\theta \in\langle 0, \varepsilon)$, for some $\varepsilon>0$, were used. Hence it is clear that one can define the notion of a small vector field and also the notions of the Kronecker index of a $(p-1)$ pseudomanifold or of a point in $\mathrm{R}^{p}$ in the same manner also for a local semi-dynamical system. It is easily verified that all the results holding for local dynamical systems also hold for local semi-dynamical systems.

Note. I have been advised that reference [2] of [6] will not be published as a separate paper, but will appear as part of chap. VI, section 3 in [5].

\section{References}

[1] Alexandroff P., Hopf H.: Topologie, Springer Verlag, Berlin 1935.

[2] Болтянский В. Г.: Гомотопическая теория непрерывных отображений и векторңых полей, Труды матем. инст. имени Стеклова XLVII, Издат. АН СССР, Москва 1955.

[3] Eilenberg S., Steenrod N. E.: Foundations of Algebraic Topology, Princeton University Press, 1952.

[4] Hájek O.: Structure of dynamical system, CMUC 6 (1965) No. 1., 53-72.

[5] Hájek O.: Dynamical Systems in the Plane. To be published by Academic Press, London.

[6] Nagy J.: Kronecker index in abstract dynamical systems, I, Czech. math. journal 15 (1965), No. 3, 394-405.

Author's address: Praha 1 - Loretánské nám. 3, ČSSR (Výzkumný ústav matematických strojů). 


\section{ИНДЕКС КРОНЕККЕРА В АБСТРАКТНЫХ ДИНАМИЧЕСКИХ СИСТЕМАХ, II}

ЙОСЕФ НАДЬ, (Jozef Nagy),Прага

В работе определяется локальная динамическая система т в $p$-мерном евклидовом пространстве $\mathrm{R}^{p}$ (определение 2.7). Потом определяется (определение 2.9 и 2.15) индекс Кронеккера ind $_{\mathrm{T}} P(p-1)$-мерного псевдомногообразия $P \subset \mathrm{R}^{p}$, не содержащего критических точек динамической системы $\mathrm{T}$, и индекс Кронеккера ind $_{\mathrm{T}} x$ точки $x \in \mathrm{R}^{p}$ относительно локальной динамической системы Т. Доказывается (теорема 2.17), что индекс ind $_{\mathrm{T}} x$ топологически инвариантен.

Дальнейшие важные результаты этой работы содержатся в следующих теоремах:

Теорема 2.10. Если $P-(p-1)$-мерное псевдомногообразие в $\mathrm{R}^{p}$ и $\overline{\operatorname{Int} P}($ замыкание внутренней области $P$ ) не содержит критических точек локальной динамической системы $\mathrm{T}$, то $\operatorname{ind}_{\mathrm{T}} P=0$.

Следствие 2.11. Если $\operatorname{ind}_{\mathrm{T}} P \neq 0$, то Int $P$ содержит по крайней мере одну критическую точку системы $\mathrm{T}$.

Теорема 2.12. Пусть ( $p-1)$-мерная сфера $S$ в $\mathrm{R}^{p}$ не содержит критических точек локальной динамической системы т. Тогда

(i) ind $_{\mathrm{T}} S=1$, если Int $S$ является - инвариантной областью,

(ii) ind $\mathrm{T} S=(-1)^{p}$, если Int $S$ является +инвариантной областью.

Следствие 2.14. Если $S-(p-1)$-мерная сфера из теоремы 2.12., то Int $S$ содержит по крайней мере одну критическую точку системы Т.

Теорема 2.16. Пусть ( $p-1)$-мерное псевдомногообразие в $\mathrm{R}^{p}$ не содержит критических точек локальной динамической системы $\mathrm{T}$ и пусть Int $P$ содержит только конечное число критических точек $x_{1}, x_{2}, \ldots, x_{n}$ системы т. Тогда имеет место соотношение

$$
\operatorname{ind}_{\mathrm{T}} P=\sum_{j=1}^{n} \operatorname{ind}_{\mathrm{T}} x_{j} \text {. }
$$

